\title{
Computer Animations, Quantum Mechanics and Elementary Particles
}

\author{
Eyal Cohen
}

ARSCIMED, Paris, France

\begin{abstract}
"A picture is worth a thousand words" is one of the rare maxims phrased as an understatement. A high-resolution colour picture contains in fact more information (bit-wise) than a million words. It is in general also faster, easier and more pleasant to grasp than mere words.
\end{abstract}

Our brain is equipped with a rapid and efficient mechanism dedicated to analysing pictures and extracting meaning from them. In spite of our tremendous handicap in arithmetic computations, we can still easily beat any computer when it comes to versatile image recognition. We are indeed so good at it that we like the exercise even better when the images are projected at the rate of at least 24 per second, in the form of video or film. For better or for worse, the dominant means of communication today is the animated image, and it is here to stay.

But there are things we, as humans, would rather express in words, and there are things - quantum mechanics and field theory among them - that we, as scientists, would express more universally in equations and numbers. This should come as no surprise, you might say: any fundamental theory is naturally presented in terms of definitions, equations and constants of nature. True: but any fundamental theory is then developed and taught with the help of examples, demonstrations, experiments, applications, and images. Practitioners of quantum mechanics somehow managed with few of the last.

The reasons for the scarcity of quantum images have very different origins, some valid, some unjustified. The following remarks illustrate the situation:

- Quantum mechanics is manifest mainly at the molecular, atomic and subatomic scales. Only recently did microscopes become capable of producing "real" images at these scales. Some isolated coherent quantum effects are known at larger scales (neutron stars, lasers, superconductivity, liquid helium, etc.), but real images demonstrating clearly their quantum aspect are lacking.

- In the domain of "theoretical" images, an overwhelming hurdle has been the rather

Eyal Cohen studied physics at Tel Aviv University and received his Ph.D. in theoretical phisics from the Wiezmann Institute, Tel Aviv, in 1982 . He was successively a postdoc at Harvard, a CERN Fellow, a postdoc at the CEA, Saclay, near Paris, and an Assistant Professor at the Wiezmann Institute. Dr. Cohen set up ARSCIMED in 1989 and remains a Scientific Counsellor at the CEA. common belief that "the quantum uncertainty principle abolished the notion of image". This is perhaps senseless. The position of an object is not a well-defined number in quantum mechanics; but a dot ("pixel") in an image does not necessarily stand for the exact position of a particle. Position and momentum cannot be simultaneously determined; but by "subtracting" an image from the following one in an animated sequence, one cannot necessarily infer momenta beyond what is allowed by the uncertainty principle. We will return to these topics in more detail.

- The objection stated above can also be heard in its "pedagogical" version: "even if a physicist can understand the subtleness of the image, a young student or a layman will be misled to believe a classical physics interpretation". In reality, the image-generation public is surprisingly sophisticated in discerning visual representations and abstractions: a subway plan, a geographical map, or an abstract painting do not need an accompanying explanation.

The almost total absence of quantum mechanics from visual culture is no doubt one reason for the general ignorance of it. For a theory, born in our century, that led to a fundamental rethinking of reality and of our perception of it, this is a rather sad state of affairs. The fundamental interactions of nature and the world of elementary particles have similarly managed to avoid major public attention, even though the accelerators dedicated to their study are among the largest and most expensive scientific laboratories in the world.

We therefore decided to develop several computer-aided visualization methods for quantum mechanics and field theories. While the public we intended to reach during the first stages of the programme was nonscientific, these methods had to have a firm scientific basis. This approach would hopefully deepen the dialogue between scientists and the public.

\section{Computer Graphics}

As the techniques of computer graphics advance rapidly, and the marvel of threedimensional imagery becomes known and affordable to an increasing number of scientists and creators in different fields, several major axes of development have emerged, including:

- realistic rendering of the visible: the creation of a virtual world almost indistinguishable from the real one;
- expressing the imagination: the computer is an almost unlimited easel;

- simulation of complicated mechanical systems, real or "virtual": designing and training to operate the real ones, or having fun in interacting with the virtual ones;

- visualizing large quantities of experimental data in an intelligent (ready for analysis) way;

- visualizing scientific models in an effort to grasp their dynamical mechanisms for research purposes, or to demonstrate the resulting phenomena for educational purposes. The methods we have created and those we wish to develop should be viewed in this context. Take high-energy physics: the experimental branch has adopted visualization as an everyday working tool whereas the theoretical branch lags behind.

\section{Quantum Mechanics}

In a first-quantised system, the classical attributes are replaced by the wave function, which is therefore the first thing one would like to visualize in a synthetic, or computergenerated image. However, when we wish to display analytical or numerical solutions of the Schrödinger equation, we encounter a major difficulty. The wave function is a complex, multi-dimensional (one function for each internal quantum number) function of a multi-dimensional coordinate space ( $\mathrm{d} N$ for $N$ particles in $d$-dimensional space) and of time. Displaying all this information on a two-dimensional screen is not an easy task. In fact, it is in general impossible: one is forced to choose a subset of the available information. Scalar fields (from now on we will use the term "function" to avoid confusion with the quantum field) are easy to display using shades of gray (typically $2^{8}$ per pixel). A possible choice of function is the electric charge density given by:

$\rho(\boldsymbol{r}, t)=\sum_{i=1}^{N} e_{i} \int(\mathrm{d} \boldsymbol{x}) \delta\left(\boldsymbol{r}-\boldsymbol{x}_{i}\right) \psi * \psi\left(\boldsymbol{x}_{1}, \ldots, \boldsymbol{x}_{N^{\prime}}, t\right)$.

(summation over internal indices is implied). replaced by other charges, or by $e_{i_{0}}=1$ to obtain the probability distribution of particle $i$. The density $\rho$ obeys the following continuity equation:

$$
\frac{\partial}{\partial \mathrm{t}} \rho(\boldsymbol{r}, t)+\Delta \cdot \boldsymbol{j}(\boldsymbol{r}, t)=0,
$$

where the current $j(r, t)$ is

$$
\begin{aligned}
& \boldsymbol{j}(\boldsymbol{r}, t)=\sum_{i=1}^{N} \frac{e_{i}}{m_{i}} \\
& \int(\mathrm{d} \boldsymbol{x}) \delta\left(\boldsymbol{r}-\boldsymbol{x}_{\mathrm{i}}\right) \operatorname{Re} \psi * \boldsymbol{p}_{\boldsymbol{i}} \psi\left(\boldsymbol{x}_{1}, \ldots, \boldsymbol{x}_{N}, t\right) .
\end{aligned}
$$


Charge conservation is expressed by:

$$
\int \rho(r, t)(\mathrm{d} r)=\sum_{i=1}^{N} e_{i} .
$$

The two-dimensional representation of a three-dimensional scalar function goes by the name "volume rendering". There are many approaches to this problem, but they fall into three main categories:

- displaying level surfaces (the set of points with a given scalar value);

- integrating function (or computing some of its moments) along rays;

- in an animation, i.e., displaying sequentially different 2-d surfaces, with colour or brightness representing the scalar value at each point.

Colour (typically $2^{24}$ values) may be used to liven up the visual aspect of the scalar function or, more interestingly, to represent internal quantum numbers or the different terms in the sum in Eq. 1, belonging to particles which are not identical. Since the individual terms are not separately conserved, the time evolution will be depicted by colour flow, as well as by brightness variations.

\section{Electron orbitals}

Consider the problem of animating the electron orbitals in the atom. The eigenstates of energy are stationary, leading to a function $\rho(r, t)$ which is time-independent. The inherent dynamics of the atom are therefore hidden when is displayed. The solution chosen was to perturb the atom by an external force, that is to say let it interact with a charged object, whose position can be identified, for example, with the incoming "camera". The cover illustration is an image from an animation following this approach. The colours correspond to different electron orbitals (assumed to be independent); the brightness is the total luminosity of sources, whose local intensity is proportional to $\rho$, positioned along lines of sight. The electron density was not obtained by a calculation of the wave function of an actual atom perturbed by an external charge, but by mapping time-dependent fractal functions on the surfaces of several spheres, conserving total probability.

Other scalar functions (such as the potential) and colour codes may be chosen; vector functions (such as $j$ ) may be displayed with the help of little arrows, etc.

In applications oriented towards the general public, the main message one is to trying to communicate by animating first-quantised systems is abolition of the classical notion of position and momentum and the probabilistic nature of the theory. The images are not only consistent with the uncertainty principle, but turn out to be extremely helpful in demonstrating and explaining it to the uninitiated among us.

\section{Quantum Field Theories}

Quantum field theory treats the properties of quantum-mechanical systems whose dynamical variables are local functions of space-time, i.e., fields whose time evolution is governed by differential equations. During second quantisation, the number of degrees of freedom becomes infinite, and an understanding of the dynamics of relevant systems such as hadrons is beyond the reach of present-day theory. The problem here is not that of visualizing otherwise known and well-understood phenomena, but grasping the fundamental mechanisms in action. For the general public, the approach here is to mainly focus on the notions of virtual particles, the quantum vacuum, and fundamental interactions.

The mental pictures that many physicists possess, and which are the predominant visual jargon for communication in discussions among them, are Feynman diagrams (see box). They are so useful in getting ideas clear for oneself, and passing them to others, that one often loses much rigour in the process. Specifically:

- Feynman diagrams only represent a perturbative expansion book-keeping device, while the theories under consideration are highly non-perturbative:

- even though the diagrams are traditionally presented in momentum space, they are often described verbally in space-time terms ("the particle enters from the right, knocks another particle, then disappears on the left...").

A useful and rather easy approach for visualizing field theories is to adopt to the letter this space-time "interpretation" of Feynman diagrams, by animating them by "throwing" point-like particles along the lines and letting the particle collide at the vertices.

\section{Lattice approach}

A more ambitious approach [1] will be described. As we shall see later, its visual aspect may be quite similar. The first step in reducing the number of degrees of freedom is to define the system on a lattice. Time may be continuous (this will, in fact, be the assumption) or discrete. In either case, an animation only samples the system at discrete moments. The approach involves associating an image to the quantum state of the system at any given time, thereby producing an animation as the system evolves in time. The tool best suited to this approach is the Hamiltonian formalism. A Hamiltonian - the transfer matrix for discrete time - is the function that, through the way it depends on its arguments, specifies the the time development of a system.

Consider the scalar field theory, defined by the continuum Langrangian:

$L=\int d^{d-1} \boldsymbol{x}\left\{\left(\frac{1}{2} \frac{\partial \varphi}{\partial t}\right)^{2}-\frac{1}{2}(\Delta \varphi)^{2}-\frac{1}{2} m^{2} \varphi^{2}-V(\varphi)\right\}$

The discrete version of the theory, on a lattice of spatial spacing a, leads to the Hamiltonian:

$$
H=\frac{1}{2} \sum_{n}\left\{a^{d-3} R(n)+a^{1-d} \pi^{2}(n)\right\},
$$

where the three-dimensional vectors $n$ correspond to the lattice sites, and

$$
\begin{aligned}
R(n)=\sum_{k=1}^{d-1}\left[\Delta_{k} \varphi(n)\right]^{2} & +m^{2} a^{2}[\varphi(n)]^{2} \\
& +2 a^{2} V(\varphi(n)) .
\end{aligned}
$$

On each lattice site, two operators ( $\phi$ and its conjugate momentum $\pi$ ) are therefore defined, such that the canonical equal-time commutation relation:

$$
\left[\pi_{t}\left(\boldsymbol{n}^{\prime}\right), \varphi_{t}(\boldsymbol{n})\right]=-\mathrm{i} \delta_{n^{\prime} n}
$$

is satisfied. The time-evolution of the system is given by the operator $\exp (-i \varepsilon H)$, where $\varepsilon$ is the time interval.

The principle of the visualization algorithm is, as in the first-quantised case, the attribution of an image to each quantum state. In the lattice formulation, the information one wishes to display is the wave function defined in terms of the local operators. Two problems are encountered, namely:

- The number of degrees of freedom per site is infinite, so what should be depicted? - Except for trivial field theories, one cannot solve the underlying equations for the quantum state of the system.

\section{Formulation}

A possible solution to the first problem is to choose a local scalar observable, such as the energy density. However, the density is poorly defined in a general quantum state, and displaying a probability distribution for each lattice site is impossible. Even expectation values are hard to compute when taking into account large numbers of degrees of freedom. The following approach is chosen instead:

1) as a first step, assign an image to only states (or rather rays) belonging to a particular complete basis, denoted by $B$;

\section{Feynman Diagrams}

Feynman diagrams (or graphs) are graphical representations of perturbationtheory calculations involving the scattering and propagation of interacting particles. In the case of relativistic quantum field theory, the particles can interact with external fields or with other particles (through the emission and absorption of virtual quanta). As an example, consider quantum electrodynamics involving electrons $e^{-}$, positrons $\mathrm{e}^{+}$, and photons $\gamma$ in the absence of external fields. The figure gives the
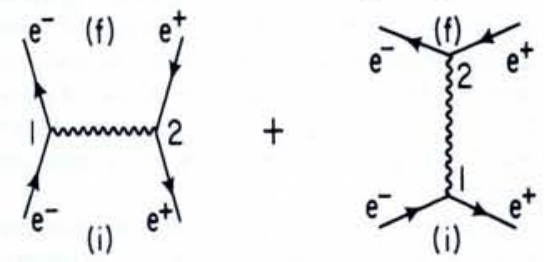

scattering amplitude for $\mathrm{e}^{+}-\mathrm{e}^{-}$scattering in lowest order: on the left, $\mathrm{e}^{+}$and $\mathrm{e}^{-}$in some initial (i) state scatter each other into a final ( $f$ ) state through the exchange of a virtual $\gamma$ which propagates to 2 and creates the final $e^{+}-e^{-}$pair. On the right, the initial $\mathrm{e}^{+}$and $\mathrm{e}^{-}$annhilate at 1 producing a virtual $\gamma$ that propagates to 2 and there produces the final $\mathrm{e}^{+}$and $\mathrm{e}^{-}$.

For calculational purposes, it is often more convenient to think of Feynman diagrams in momentum rather than coordinate space. In either case, incoming and outgoing particles are represented as external lines and Feyman propagators or Green's function as internal lines. Rules associate specific functions with each line and vertex, specify sign conventions and, in momentum space, require conservation of energy and momentum at each vertex. 
2) define the local operator to be displayed, and how to form an image given its expectation value as a function of space;

3) truncate the basis where additional states would not be visible;

4) the system evolves solely by the operator $\exp (-i \varepsilon H)$.

A "picture" of the quantum state is "taken" at regular time intervals, projecting it to the chosen basis. The probability to evolve from the state $i$ at time $t$ to the state $i^{\prime}$ at time $t+\varepsilon$ is given by:

Prob. $\left[\left(i_{t+\varepsilon}\right) /\left(i_{t}\right)\right]=\left|\left\langle i_{t+\varepsilon}^{\prime} \mid \exp (-i \varepsilon H) i_{t}\right\rangle\right|^{2}$.

If one is smart enough to choose the par ticular basis as eigenstates of the Hamiltonian (physical states), they will remain so as time evolves. This, however, amounts to solving the theory. In practice, one should try an ansatz as close to the physical states as possible. This would allow the animation of phenomena such as scattering, radioactive decay, etc.

As long as the chosen basis is not physical, frequent measurements on the system may destroy coherence effects. The importance of these effects depends on the theo$\mathrm{ry}$, and on the choice of states and approximations: their sensitivities can be tested by varying the measurement frequency. If coherence turns out to be crucial, the first of the above rules must be relaxed somewhat to allow the superposition of basis states, up to a certain level. Pictorially, this can be represented by the superposition of images, although phases will be lost.

A useful feature of the formulation is that animations can be performed even when nothing is known about the physical properties of the theory, apart from its Hamiltonian. While the value of these animations as simulations of physical systems is very limited in these cases, the animations usually turn out to be a useful popularisation and pedagogical tool: they may even lead to further insight. The result will in the end resemble the Feynman diagram approach.

The operators $\phi(n)$ and $\pi(n)$ in the continuum Langrangian may be expressed in terms of the creation and annihilation operators as follows:

$$
\begin{aligned}
& \varphi(n)=\frac{1}{\sqrt{2 m}} a^{(1-d) / 2}\left(a^{+}(n)+a(n)\right), \\
& \pi(n)=\sqrt{ } \frac{m}{2} a^{(d-1) / 2} i\left(a^{+}(n)-a(n)\right), \\
& {\left[a\left(n^{\prime}\right), a^{+}(n)\right]=\delta_{n^{\prime} n^{n}}}
\end{aligned}
$$

The visualization basis will be chosen as the set of states:

$$
\prod_{n} \frac{1}{\sqrt{ } i_{n} !\left(a^{+}(n)\right)^{i n} \mid 0>,}
$$

$i_{n}$ being a set of non-negative integers, one for each lattice site.

The operators we choose to display are the local number operators $a^{+} a(n)$. For any of the basis states, this number is an integer, to be interpreted as the number of objects to be displayed. The shape of the objects, as well as their "optical" properties with respect to the "light" sources which illuminate the scene in the computer-generated image (colour, transparency, reflection, and absorption coefficients, etc.), may be used to distinguish among the values of the internal quantum numbers that have not been specified.

\section{Results and renormalization}

The Hamiltonian describes a system of non-harmonic oscillators (one per site) where the kinetic term couples nearestneighbour sites. For values of the time interval small enough to allow the use of firstorder perturbation theory, the kinetic term will cause the particles to diffuse to nearestneighbour sites. The interaction term will lead to vertices involving several particles at a time, including the creation and annihilation of particles. In short, the animations are vivid Feynman diagrams, with the particles following Brownian motion and not straight paths, due to the uncertainty in momentum

An interesting visual effect is obtained when the shutter delay of the "camera" is chosen to be several times the frame interval $\varepsilon$. This allows particles to be present in the frame even after their annihilation, and therefore each one of the still pictures has the Feynman diagram "look".

In a typical animation, starting from a small number of virtual particles, the number tends to increase as a function of time, signalling the deviation from the physical states. A physical particle contains a cloud of finite size of virtual particles. The animations actually allow us to see the formation of such clouds. It is rather amusing to identify dressed objects manifesting collective behavior, and then analyse the space renormalization group structure of the clouds by zooming in.

\section{Quantum Chromodynamics}

So far we have described the animation of scalar field theories, but the most interesting application of the method involves the strong interactions. In principle, one can follow the same formulation. The QCD lattice Hamiltonian was developed by Kogut and Susskind [2], and applied mainly to strong coupling approximations. It possesses the convenient property that confinement is manifest in the strong coupling limit. To animate processes such as scattering, one should look for physical states, which in this case are also gauge-invariant. In practice, this is a formidable task, and one is necessarily led to the type of perturbative treatment discussed above, with the virtual particles being bare quarks and gluons: the quarks live on the lattice sites, and the gluons on the links.

It is interesting to mention recent work by Llewellyn Smith and Watson [3], who applied the coupled-cluster method to lattice (pure) gauge theories, and obtained good results for the clustered (localised) wave functions of the vacuum as well as excited states. This result is encouraging when one pursues, as is done here, an inherently local procedure.

Owing to limited computational resources (most of the computations were performed part-time on a 4D20 SGI workstation), and the need to produce animations in a reasonable amount of time and at reasonable cost (less than an hour of computer time per video frame), fully-fledged QCD could not be tackled. A field theory was selected instead, where both quarks and gluons are scalar fields, and where their interaction vertices are the same as in QCD. In order to emulate confinement, the coupling constant at any given site was chosen to be a function of the distance to a number of external colour sources (three for the animation of the inside of the proton). The particles possess a conserved "colour" quantum number, corresponding to the Lie group

$$
\frac{U_{(1) \text { red }} \times U_{(1) \text { green }} \times U_{(1) \text { blue }}}{U_{(1) \text { red }+ \text { green+blue }}}
$$

The number of virtual particles that can be followed in the course of the animation is

\section{Postdoctoral Fellowships}

at the

\section{Niels Bohr Institute and Nordita}

The Niels Bohr Institute and Nordita invite applications for postdoctoral fellowships in physics.

The Niels Bohr Institute is part of the physics department of Copenhagen University and has active research programs in theoretical and experimental particle physics, theoretical and experimental nuclear physics, physics of nonlinear and complex systems, cluster physics and theoretica and observational astrophysics. The Institute operates or has access to research facilities such the NORDBALL detector. CERN accelerators, ESO, NOT and satellite telescopes, cluster physics facilities and a range of computers.

Applicants for positions at the Niels Bohr Institute who are citizens and residents of EC member states (or countries treated as member states) are eligible for fellowships at the EC Physics Training Centre at the Niels Bohr Institute. This is supported by the European Community "Human Capital and Mobility" program, and the stipends set by the EC rules. Applicants will also be considered under the Institute's OWII international programs.

Nordita, the Nordic Institute for Theoretical Pllysics, is supported by the governments of Denmark Finland, Iceland, Norway and Sweden. It is located within the same premises as the Niels Boh Institute, and has theoretical research programs in astrophysics, cluster physics, condensed matter physics, the physics of nonlinear and complex systems, nuclear physics and particle physics Positions are open to scientists independent of nationality. Scientists from Nordita member countries are also eligible for Nordita Fellowships, wlich must be applied for separately: information about these may be obtained from Nordita.

Applicants should include a curriculum vitae, list of publications and a statement of their research interests and goals. In addition, applicants should arrange for 2-3 letters of reference to be sent directly. All material should be sent to:

NBI/Nordita Postdoctoral Positions Office,

Ulla Holm, secretary, Blegdamsvej 17, DK-2100 Copenhagen, Denmark.

The deadline for applications for 1993/94 is 15 December 1992.

Niels Bohr Institute: Tel.: (+45) 314216 16; Fax: (+45) 31421016 ; E-mail: uhoim@nbivax.nbi.dk Nordita: Tel.: (+45) 314216 16; Fax: (+45) 31389157 E-mail: nordita@nordita.dk 
limited by computing time to about 2000: the total number of interaction events is approximately 100000 .

\section{Confinement and renormalization}

Fig. 1 shows the way confinement works in the model. A quark and an antiquark were separated with opposite initial velocities. Virtual quarks, antiquarks (both represented by spheres), and gluons (represented by elongated ellipsoids) are emitted from the vacuum and a flux tube is formed.

Exploration of the space renormalization group structure of an image requires more than several thousands of particles so this cannot be done with the animations generated so far. However, more computing time can be afforded for the creation of still pictures, since fewer are needed. The renormalization group idea described above was therefore applied to still images. The procedure is the following:

1) start from any of the animated configurations (containing, for example, several hundreds of virtual particles);

2) choose geometrical shapes for dressed constituent objects (spherical for quarks and antiquarks, cylindrical for flux lines were chosen in the present case);

3) dress each one of the objects in the animated configuration by a collection (on the order of ten) of the above geometrical shapes with variable sizes, such that the resulting overall shape is also spherical or cylindrical.

4) repeat the procedure for each one of the components of the cloud.

Starting from the animation of the generation of a proton from three valence quarks, Fig. 2 shows the result of this repeated "fractalization" process, applied four times. The total number of particles is around 50000 . The distribution of the constituents in the cloud at each step is random in Fig. $2 a$, while in Fig. $2 b$ it is similar (up to a scale transformation) to that obtained in the previous step.

Based on the "look" of the nucleon in the images of Fig. 2, and using standard rende-

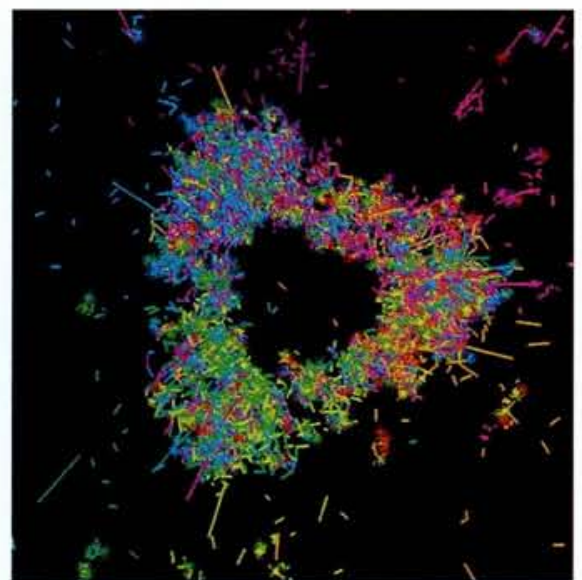

gned to a slice of space, will work in paralle for the generation of each frame, thereby reducing significantly the computing time per frame for a given field theory, allowing the exploration of more complex theories.

\section{Conclusions}

The visualization "dictionary" developed for computer animations of quantum systems can be applied to any process following the rules of one or several of Nature's fundamental interactions. Animation of various atomic and subatomic phenomena, such as electron orbitals, particle collisions radioactive decay, fusion, fission, etc. are therefore feasible and instructive.

Films are being used to illustrate televi-

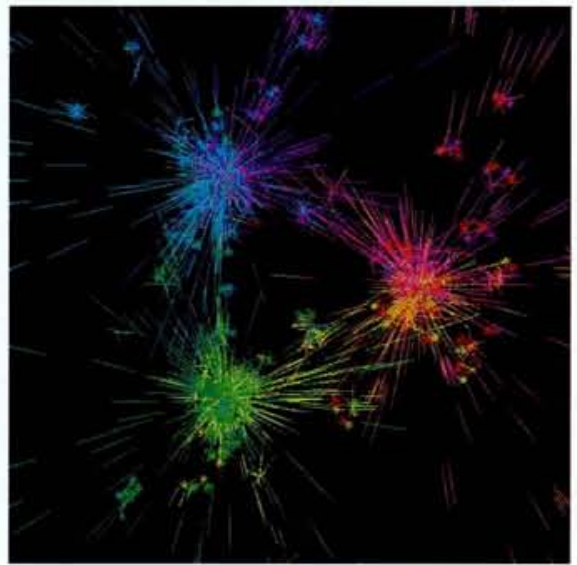
sion programmes and to demonstrate the physics and visual concepts involved, as well as in museums and education. The hope is that that with computer parallelization and access to more powerful computers, a research tool useful in the hands (or rather in the eyes) of physicists can also be developed. Only time will tell whether a deeper understanding of present day theories, and inspiration for new ones, will be directly attributable to the force of the image.

\section{REFERENCES \& FURTHER INFORMATION}

[1] Cohen E., Comp. Phys. Com. 70 (1992) 441.

[2] Kogut J. and Susskind L., Phys. Rev. D 11 (1975) 395.

Fig. 2 - Snapshots of the process of hadronization during the generation of a proton from three valence quarks: $a$, upper) the fractionalization process; $b$, lower) including the interaction.

ring tools (e.g., the program ALIAS), images of several different nuclei were created, including that of silicon $\left({ }_{14} \mathrm{Si}^{28}\right)$ shown in Fig. 3.

A parallel version of the algorithm is presently being developed to attack real QCD calculations. Several processors, each assi-
[3] Watson N.J., Coupled Cluster Methods in Lattice Gauge Theory (Ph.D. Thesis, Oxford University, UK) 1992; Llewellyn Smith C. (private communication).

Several video programs including animations of the proton, the pion and the atom using the methods outlined in this article, as well as still pictures, are available. For further information contact:

Eyal Cohen, ARSCIMED (art, science, media) 141 , bis quai de Valmy, F-75010 Paris (tel: +33 (1) 460709 49; fax: +33 (1) 40383743 ).
Fig. $1-A$ demonstration of confinement showing a flux tube that is formed when a source of colour is separated from a source of anticolour. Virtual quarks, antiquarks (both represented by spheres) and gluons (represented by elongated ellipsoids) are emitted from the vacuum.

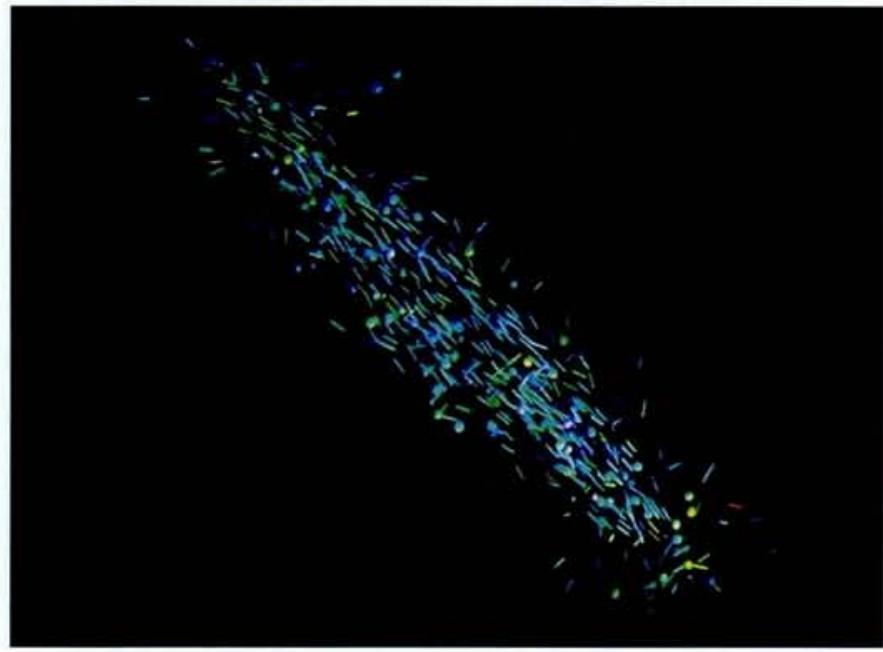

Fig. 3 - The constituent dressed quarks inside the protons and neutrons that make up the silicon ${ }_{14} \mathrm{Si}^{28}$ nucleus. Commercial computerbased rendering techniques have been used to stimulate the virtual particle structures of the objects based on the "look" of the nucleon shown in Fig. 2.

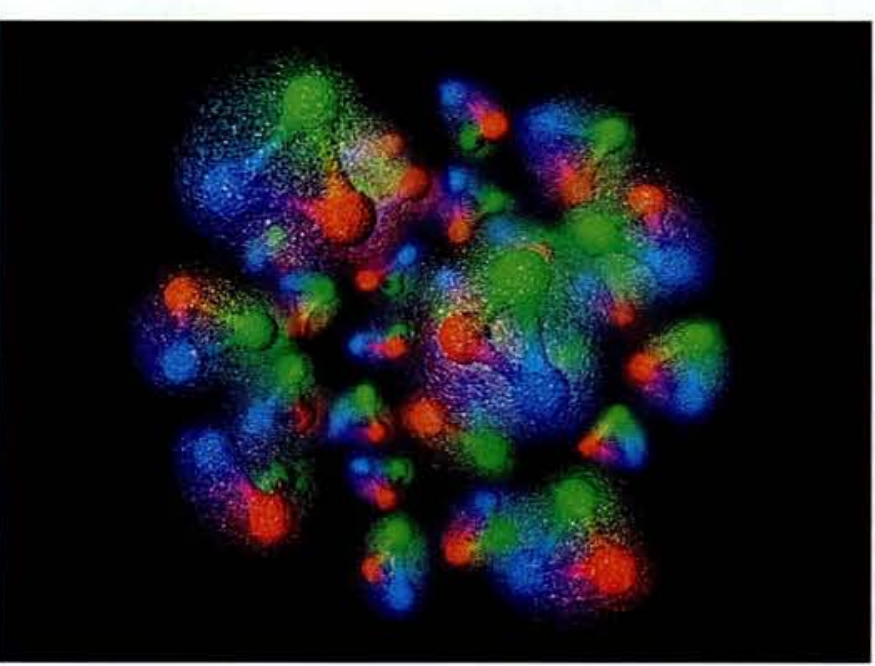

